\title{
A Derivation of the Ricci Flow
}

\author{
Vu B. Ho \\ 9 Adela Court, Mulgrave, Australia \\ Email: vubho@bigpond.net.au
}

How to cite this paper: Ho, V.B. (2021) A Derivation of the Ricci Flow. Journal of Applied Mathematics and Physics, 9, 2179-2186. https://doi.org/10.4236/jamp.2021.99137

Received: July 31, 2021

Accepted: August 31, 2021

Published: September 3, 2021

Copyright (C) 2021 by author(s) and Scientific Research Publishing Inc. This work is licensed under the Creative Commons Attribution International License (CC BY 4.0).

http://creativecommons.org/licenses/by/4.0/

\begin{abstract}
In this work, we show that by restricting to the subgroup of time-independent coordinate transformations, then it is possible to derive the Ricci flow from the Bianchi identities. To achieve this, we first show that the field equations of the gravitational field, the Newton's second law of classical dynamics, and the Maxwell field equations of the electromagnetic field all share the same mathematical structure. Consequently, the Ricci flow itself may be regarded as dynamical equations used to describe physical processes associated with the gravitational field, such as the process of smoothing out irregularities of distribution of matter in space.
\end{abstract}

\section{Keywords}

Ricci Flow, Bianchi Identities, General Relativity, Classical Physics

\section{Introduction}

Geometrical and topological methods have played an important role in the formulations of physical theories that characterise the quantum nature of physical entities in terms of mathematical objects [1] [2] [3] [4]. These formulations can be extended to show that quantum particles may in fact manifest as three-dimensional differentiable manifolds whose spacetime structures are governed by the field equations of general relativity which, as shown below, can be formulated purely in terms of differential geometry [5]-[12]. In general, the geometrical approach to formulating fundamental structures of quantum particles requires the construction of a space so that physical objects can be identified with the geometrical properties of that space. The physical description associated with the geometrical structures is expressed in the form of field equations, such as Einstein's field equations of general relativity [13]. Geometrical and topological methods applied to physical theories are a way of establishing a correspondence between mathematical concepts and physical objects. This can be achieved by searching 
for mathematical structures that can provide a consistent description of the dynamics of physical observables. Conversely, physical objects may provide an empirical realisation of the abstract concepts associated with geometry and topology. However, there are restrictions on the applicability of geometrical and topological methods in the construction of physical theories. A general feature of geometrical models applied to physics is that their mathematical realisation can only be described approximately since physical theories have been based on concepts, such as mass, which are poorly defined from the point of view of mathematics. Geometrical procedures that require the generalisation of such concepts may also lead to a poor description of physical phenomena. For this reason, Einstein's theory of general relativity cannot be a truly geometrical theory, because the energy-momentum tensor which enters the theory is a non-geometrical quantity [14]. However, this blemish can be overcome by formulating the field equations of the gravitational field using the contracted Bianchi identities in which Einstein field equations of general relativity are considered, as in the case of the electromagnetic field, as a definition of the energy-momentum tensor for the gravitational field.

\section{Covariant Formulation of Classical Physics}

Even though the focus of this work is to derive the Ricci flow from our formulation of the gravitational field from the Bianchi identities, it is noted that classical physics, which includes Newton dynamics, Maxwell field equations of electromagnetism and Einstein general relativity, can be formulated from a general equation in which the mathematical objects that are used to formulate these physical theories differ only by their mathematical natures. Consider an equation of the general form

$$
\nabla_{\beta} \mathrm{M}=\mathrm{J}
$$

where $\mathrm{M}$ is a mathematical object, $\mathrm{J}$ a physical entity, and $\nabla_{\beta}$ a covariant derivative. In fact, we may speculate that the equation given in Equation (1) can be applied to any physical field by establishing a suitable mathematical object for $\mathrm{M}$ and a physical object for J. For Newton dynamics in Euclidean space, we set $\mathrm{M}=E$ with

$$
E=\frac{1}{2} m \sum_{\mu=1}^{3}\left(\frac{\mathrm{d} x^{\mu}}{\mathrm{d} t}\right)^{2}+V
$$

In this case, the mathematical object $M$ given in Equation (1) is a scalar, and with $J=0$ the dynamical equation given in Equation (1) reduces to Newton's second law

$$
\frac{\partial E}{\partial x^{\mu}}=0
$$

For Maxwell field equations of electromagnetism in Euclidean space, we set $\mathrm{M}=F^{\alpha \beta}$, where the electromagnetic tensor $F^{\alpha \beta}$ expressed in terms of the four-vector potential $A^{\mu} \equiv(V, \mathbf{A})$ as 


$$
F^{\alpha \beta}=\frac{\partial A^{\beta}}{\partial x^{\alpha}}-\frac{\partial A^{\alpha}}{\partial x^{\beta}}
$$

In this case, $\mathrm{M}$ is an anti-symmetric tensor and the dynamical equation given in Equation (1) reduces to Maxwell field equations

$$
\frac{\partial F^{\alpha \beta}}{\partial x^{\alpha}}=\mu j^{\beta}
$$

in which the four-current $j^{\beta} \equiv\left(\rho_{e}, \mathbf{j}_{e}\right)$. From the fact that the electromagnetic tensor $F^{\alpha \beta}$ is anti-symmetric, it can be expressed as a dual vector. In electromagnetism, the electromagnetic energy-momentum tensor $T^{\alpha \beta}$ for the free electromagnetic field with the defined Lagrangian of the form $L=-\frac{1}{4} F^{\alpha \beta} F_{\alpha \beta}$ can be established as

$$
T^{\alpha \beta}=\epsilon_{0} c^{2}\left(\eta_{\mu v} F^{\alpha \mu} F^{\nu \beta}+\frac{1}{4} \eta^{\alpha \beta} F_{\lambda v} F^{\lambda v}\right)
$$

where $\eta_{\alpha \beta}$ is the Minkowski metric tensor [15].

Now, to use the general equation given in Equation (1) to represent the gravitational field, we would need to reinterpret Einstein field equations of general relativity so that the overall mathematical structure of the gravitational field would look like that of the electromagnetic field, with the field equations given by Equation (5) and the energy-momentum tensor given by Equation (6). It is shown in differential geometry that the Ricci tensor $R^{\alpha \beta}$ satisfies the Bianchi identities [16]

$$
\nabla_{\beta} R^{\alpha \beta}=\frac{1}{2} g^{\alpha \beta} \nabla_{\beta} R
$$

where the Ricci tensor $R_{\alpha \beta}$ defined in terms of the affine connection $\Gamma_{\alpha \beta}^{\gamma}$ as

$$
R_{\alpha \beta}=\frac{\partial \Gamma_{\alpha \beta}^{\sigma}}{\partial x^{\sigma}}-\frac{\partial \Gamma_{\alpha \sigma}^{\sigma}}{\partial x^{\beta}}+\Gamma_{\alpha \beta}^{\lambda} \Gamma_{\lambda \sigma}^{\sigma}-\Gamma_{\alpha \sigma}^{\lambda} \Gamma_{\lambda \beta}^{\sigma}
$$

To formulate the field equations for the gravitational field it is necessary to introduce a metric tensor $g_{\alpha \beta}$ in terms of which the affine connection $\Gamma_{\alpha \beta}^{\gamma}$ is defined

$$
\Gamma_{\alpha \beta}^{\lambda}=\frac{1}{2} g^{\lambda \sigma}\left(\frac{\partial g_{\sigma \beta}}{\partial x^{\alpha}}+\frac{\partial g_{\sigma \alpha}}{\partial x^{\beta}}-\frac{\partial g_{\alpha \beta}}{\partial x^{\sigma}}\right)
$$

Even though Equation (7) is purely geometrical, it has a form like Equation (5) for the electromagnetic tensor. Therefore, we may identify $M$ in Equation (1) with the Ricci tensor $\mathrm{M}=R^{\alpha \beta}$. And in this case, the quantity $\mathrm{J}$ in Equation (1) is defined purely in terms of geometrical objects as

$$
j^{\alpha}=\frac{1}{2} g^{\alpha \beta} \nabla_{\beta} R
$$

If the quantity $\frac{1}{2} g^{\alpha \beta} \nabla_{\beta} R$ can be determined as a physical entity, such as a four-current of the gravitational matter, then Equation (7) has the status of a 
dynamical law of a physical theory which can be postulated as the field equations of the gravitational field. In this case, as in the case of the electromagnetic field, the energy-momentum tensor $T_{\alpha \beta}$ for the gravitational field can be established in terms of the Ricci tensor $R_{\alpha \beta}$ and the metric tensor $g_{\alpha \beta}$ as

$$
T_{\alpha \beta}=k\left(R_{\alpha \beta}-\frac{1}{2} g_{\alpha \beta} R\right)
$$

Equation (11) is Einstein field equations of general relativity. For a purely gravitational field in which $\frac{1}{2} g^{\alpha \beta} \nabla_{\beta} R=0$, Equation (7) reduces to

$$
\nabla_{\beta} R^{\alpha \beta}=0
$$

Solutions, such as the Schwarzschild solution, can be obtained from Equation (12) by observing that since $\nabla_{\mu} g^{\alpha \beta} \equiv 0$ we have

$$
R_{\alpha \beta}=\Lambda g_{\alpha \beta}
$$

where $\Lambda$ is an undetermined constant. For $\Lambda=0$, and if we consider a centrally symmetric gravitational field with the line element [17] [18]

$$
\mathrm{d} s^{2}=\mathrm{e}^{\psi} c^{2} \mathrm{~d} t^{2}-\mathrm{e}^{\chi} \mathrm{d} r^{2}-r^{2}\left(\mathrm{~d} \theta^{2}+\sin ^{2} \theta \mathrm{d} \phi^{2}\right)
$$

then the Schwarzschild solution can be found as

$$
\mathrm{d} s^{2}=\left(1-\frac{2 G M}{r}\right) c^{2} \mathrm{~d} t^{2}-\left(1-\frac{2 G M}{r}\right)^{-1} \mathrm{~d} r^{2}-r^{2}\left(\mathrm{~d} \theta^{2}+\sin ^{2} \theta \mathrm{d} \phi^{2}\right)
$$

\section{Derivation of Ricci Flow}

In this section, we derive from Equation (12) the intrinsic geometric Ricci flow introduced by Hamilton [19] [20], and given as follows

$$
\frac{\partial g_{\alpha \beta}}{\partial t}=-2 R_{\alpha \beta}
$$

Mathematically, the Ricci flow given in Equation (16) is a geometric process that can be employed to smooth out irregularities of a Riemannian manifold. In general, the partial time derivative of a tensor is not a tensor therefore the Ricci flow is not a tensorial equation. However, Equation (16) becomes a tensorial equation with respect to the group of time-independent coordinate transformations. This is in fact an important feature because we can derive the Ricci flow within this subgroup of coordinate transformations. In differential geometry, the covariant derivative of a contravariant tensor of second rank $A^{\alpha \beta}$ is given by

$$
\nabla_{\gamma} A^{\alpha \beta}=\partial_{\gamma} A^{\alpha \beta}+\Gamma_{\sigma \gamma}^{\alpha} A^{\sigma \beta}+\Gamma_{\sigma \gamma}^{\beta} A^{\alpha \sigma}
$$

The partial time derivative of Equation (17) is

$$
\begin{aligned}
\partial_{t}\left(\nabla_{\gamma} A^{\alpha \beta}\right)= & \partial_{t}\left(\partial_{\gamma} A^{\alpha \beta}\right)+\left(\partial_{t} \Gamma_{\sigma \gamma}^{\alpha}\right) A^{\sigma \beta}+\Gamma_{\sigma \gamma}^{\alpha}\left(\partial_{t} A^{\sigma \beta}\right) \\
& +\left(\partial_{t} \Gamma_{\sigma \gamma}^{\beta}\right) A^{\alpha \sigma}+\Gamma_{\sigma \gamma}^{\beta}\left(\partial_{t} A^{\alpha \sigma}\right)
\end{aligned}
$$

Under the coordinate transformation $x^{\prime \alpha}=f^{\alpha}\left(x^{\beta}\right)$, the tensor $A^{\alpha \beta}$ is transformed as 


$$
A^{\prime \alpha \beta}=\frac{\partial x^{\prime \alpha}}{\partial x^{\rho}} \frac{\partial x^{\prime \beta}}{\partial x^{\sigma}} A^{\rho \sigma}
$$

If the coordinate transformation is time-independent then the partial time derivative of the tensor $A^{\alpha \beta}$ is also a tensor which is transformed according to the rule

$$
\frac{\partial A^{\prime \alpha \beta}}{\partial t}=\frac{\partial x^{\prime \alpha}}{\partial x^{\rho}} \frac{\partial x^{\prime \beta}}{\partial x^{\sigma}} \frac{\partial A^{\rho \sigma}}{\partial t}
$$

In this case, we have

$$
\nabla_{\gamma}\left(\partial_{t} A^{\alpha \beta}\right)=\partial_{\gamma}\left(\partial_{t} A^{\alpha \beta}\right)+\Gamma_{\sigma \gamma}^{\alpha}\left(\partial_{t} A^{\sigma \beta}\right)+\Gamma_{\sigma \gamma}^{\beta}\left(\partial_{t} A^{\alpha \sigma}\right)
$$

It is observed from Equations (18) and (21) that if we impose the following condition on Equation (18)

$$
\left(\partial_{t} \Gamma_{\sigma \gamma}^{\alpha}\right) A^{\sigma \beta}+\left(\partial_{t} \Gamma_{\sigma \gamma}^{\beta}\right) A^{\alpha \sigma}=0
$$

then we obtain the identity

$$
\nabla_{\gamma}\left(\partial_{t} A^{\alpha \beta}\right)=\partial_{t}\left(\nabla_{\gamma} A^{\alpha \beta}\right)
$$

For a metric tensor $g^{\alpha \beta}$ we have $\nabla_{\gamma}\left(\partial_{t} g^{\alpha \beta}\right)=\partial_{t}\left(\nabla_{\gamma} g^{\alpha \beta}\right) \equiv 0$, and in this case from the field equations given in Equation (12), $\nabla_{\beta} R^{\alpha \beta}=0$, we arrive at

$$
\frac{\partial g_{\alpha \beta}}{\partial t}=k R_{\alpha \beta}
$$

where $k$ is a scaling factor. As an example, consider a simple line element of the form

$$
\mathrm{d} s^{2}=D(c \mathrm{~d} t)^{2}-A(x, y, z, t)\left[(\mathrm{d} x)^{2}+(\mathrm{d} y)^{2}+(\mathrm{d} x)^{2}\right]
$$

where $D$ is constant. If the coordinate transformations are time-independent then the imposed conditions $\left(\partial_{t} \Gamma_{\alpha \gamma}^{\sigma}\right) g_{\sigma \beta}+\left(\partial_{t} \Gamma_{\beta \gamma}^{\sigma}\right) g_{\alpha \sigma}=0$ lead to the following system of equations for the quantity $A$

$$
\begin{gathered}
\frac{\partial^{2} A}{\partial t^{2}}=0 \\
\frac{\partial^{2} A}{\partial t^{2}}-\frac{1}{A}\left(\frac{\partial A}{\partial t}\right)^{2}=0 \\
\frac{\partial^{2} A}{\partial t \partial x}-\frac{1}{A}\left(\frac{\partial A}{\partial t}\right)\left(\frac{\partial A}{\partial x}\right)=0 \\
\frac{\partial^{2} A}{\partial t \partial y}-\frac{1}{A}\left(\frac{\partial A}{\partial t}\right)\left(\frac{\partial A}{\partial y}\right)=0 \\
\frac{\partial^{2} A}{\partial t \partial z}-\frac{1}{A}\left(\frac{\partial A}{\partial t}\right)\left(\frac{\partial A}{\partial z}\right)=0
\end{gathered}
$$

It is seen from the above conditions that the quantity $A$ is also time-independent and the Ricci flow given in Equation (24) leads to the purely gravitational field $R_{\alpha \beta}=0$ which admits only time-independent solutions. Since $R_{\alpha \beta}=0$ implies $R=0$, we obtain the following equation for the quantity $A$ 


$$
\nabla^{2} A+\frac{3}{4 A}(\nabla A)^{2}=0
$$

We would like to make a remark here that since the Ricci flow given in Equation (24) is derived from Equation (12) which is assumed to be field equations that describe the gravitational field, therefore it is reasonable to suggest that the Ricci flow itself may also be regarded as dynamical equations that can be used to describe physical processes associated with the gravitational field, such as the process of smoothing out irregularities of distribution of matter in space.

\section{Further Discussion}

It was mentioned above that the Ricci flow given in Equation (24) is a tensorial equation only for time-independent coordinate transformations. However, a general tensorial Ricci flow can be formulated using the Lie derivatives. In differential geometry, the Lie derivative of a general tensor field $T_{\beta \ldots}^{\alpha \ldots}$ is given as [16]

$$
L_{X} T_{\beta \ldots}^{\alpha \ldots}=X^{\mu} \partial_{\mu} T_{\beta \ldots}^{\alpha \ldots}-T_{\beta \ldots}^{\mu \ldots} \partial_{\mu} X^{\alpha}-\cdots+T_{\mu \ldots}^{\alpha \ldots} \partial_{\beta} X^{\mu}+\cdots
$$

For the case of a covariant metric tensor $g_{\alpha \beta}$ we have

$$
L_{X} g_{\alpha \beta}=X^{\mu} \partial_{\mu} g_{\alpha \beta}+g_{\alpha \mu} \partial_{\beta} X^{\mu}+g_{\beta \mu} \partial_{\alpha} X^{\mu}
$$

Besides the important properties of being linear, satisfying the product rule for differentiation and commuting with contraction, the Lie differentiation with respect to a vector field $X$ also preserves the type of a tensor. Since the Lie derivative of a covariant metric tensor of second rank is also a covariant tensor of second rank we may propose the following tensor equation

$$
L_{X} g_{\alpha \beta}=k R_{\alpha \beta}
$$

where $k$ is a dimensional constant. Using Equation (33), Equation (34) becomes

$$
X^{\mu} \partial_{\mu} g_{\alpha \beta}+g_{\alpha \mu} \partial_{\beta} X^{\mu}+g_{\beta \mu} \partial_{\alpha} X^{\mu}=k R_{\alpha \beta}
$$

Mathematically, the covariant flow given in Equation (35) can be reduced to the Ricci flow given in Equation (24) if the vector field $X^{\mu}$ can be smoothly assigned values in the form $X^{\mu}=\left(X^{0}, 0,0,0\right)$, where $X^{0}$ is a constant temporal component of the vector field. In this case we obtain the form of the Ricci flow given in Equation (24) as

$$
X^{0} \partial_{0} g_{\alpha \beta}=k R_{\alpha \beta}
$$

Physically, Equation (36) can be explained using commoving synchronous coordinate systems on a homogeneous three-dimensional spatial manifold $S$ formed by some fluid substance [17].

\section{Conclusion}

We have shown that Newton dynamics, Maxwell field equations of electromagnetism, and Einstein general relativity all share the same mathematical structure given by the general equation $\nabla_{\beta} \mathrm{M}=\mathrm{J}$ in which the mathematical objects used to formulate these physical theories differ only by their mathematical na- 
tures. We speculate that this equation may also be applied to any physical field by defining a suitable mathematical object for $\mathrm{M}$ and a physical object for $\mathrm{J}$. Then, we have shown that by restricting the coordinate transformations to those that are time-independent, it is possible to derive the Ricci flow from the Bianchi identities, which in turns are postulated as field equations for the gravitational field. Consequently, the Ricci flow itself may be regarded as dynamical equations used to describe physical processes associated with the gravitational field, such as the process of smoothing out irregularities of distribution of matter in space.

\section{Acknowledgements}

We would like to thank the reviewers for their constructive comments, and we would also like to thank the administration of JAMP for their editorial advice during the preparation of this work.

\section{Conflicts of Interest}

The author declares no conflicts of interest regarding the publication of this paper.

\section{References}

[1] Balachandran, A.P., Marmo, G., Skagerstam, B.S. and Stern, A. (1991) Classical Topology and Quantum States. World Scientific, Singapore. https://doi.org/10.1142/1180

[2] Kauffman, L.H. (2001) Knots and Physics. World Scientific, Singapore. https://doi.org/10.1142/4256

[3] Ho, V.B. (1994) On the Quantization of Angular Momentum. Journal of Physics A: Mathematical and General, 27, 6237-6241. https://doi.org/10.1088/0305-4470/27/18/031

[4] Ho, V.B. and Morgan, M.J. (1996) Quantum Mechanics in Multiply-Connected Spaces. Journal of Physics A: Mathematical and General, 29, 1497-1510. https://doi.org/10.1088/0305-4470/29/7/019

[5] Ho, V.B. (1996) Geometrical and Topological Methods in Classical and Quantum Physics. PhD Thesis, Monash University, Clayton.

[6] Ho, V.B. (2018) Spacetime Structures of Quantum Particles. International Journal of Physics, 6, 105-115. https://doi.org/10.12691/ijp-6-4-2

[7] Ho, V.B. (2018) A Classification of Quantum Particles. GJSFR-A, 18, 37-58.

[8] Ho, V.B. (2018) Quantum Particles as 3D Differentiable Manifolds. International Journal of Physics, 6, 139-146. https://doi.org/10.12691/ijp-6-5-1

[9] Ho, V.B. (2018) Fluid State of Dirac Quantum Particles. Journal of Modern Physics, 9, 2402-2419. https://doi.org/10.4236/jmp.2018.914154

[10] Ho, V.B. (2018) Topological Transformation of Quantum Dynamics. Journal of Modern Physics, 10, 102-127. https://doi.org/10.4236/jmp.2019.102009

[11] Ho, V.B. (2018) Fluid State of the Electromagnetic Field.

[12] Ho, V.B. (2020) Classification of Relativity. Journal of Modern Physics, 11, 535-564. https://doi.org/10.4236/jmp.2020.114036 
[13] Einstein, A. (1952) The Principle of Relativity. Dover Publications, New York.

[14] Hawking, S.W. (1979) General Relativity, an Einstein Centenary Survey. Cambridge University Press, Cambridge.

[15] Landau, L.D. and Lifshitz, E.M. (1975) The Classical Theory of Fields. Pergamon, Sydney.

[16] D’Inverno, R. (1992) Introducing Einstein' Relativity. Clarendon Press, Oxford.

[17] Misner, C.W., Thorne, K.S. and Wheeler, J.A. (1971) Gravitation. W. H. Freeman and Company, New York.

[18] Kramer, D., Stephanie, H., Hert, E. and MacCallum, M. (1980) Exact Solutions of Einstein's Field Equations. Cambridge University Press, Cambridge.

[19] Hamilton, R.S. (1982) Three-Manifolds with Positive Ricci Curvature. Journal of Differential Geometry, 17, 255-306. https://doi.org/10.4310/jdg/1214436922

[20] Cao, H.-D. and Zhu, X.-P. (2006) A Complete Proof of the Poincaré and Geometrization Conjectures-Application of the Hamilton-Perelman Theory of the Ricci Flow. The Asian Journal of Mathematics, 10, 165-492.

https://doi.org/10.4310/AJM.2006.v10.n2.a2 